\title{
Temporal changes in the genetic structure of Daphnia magna populations
}

\author{
Helena Korpelainen
}

Department of Genetics, University of Helsinki, Arkadiankatu 7, SF-00100 Helsinki 10, Fin!and

\begin{abstract}
Successive sampling of temporary Daphnia magna populations revealed significant fluctuations in genotype and allele frequencies, but these changes did not show any clear seasonal pattern. Rapid frequency changes, which were mostly unique for given populations, reflect differences in fitness values between genotypic classes. It is suggested that the effect of selection is strengthened by the lowered effective population sizes due to great fecundity differentials and fluctuating population sizes. Specially great genetic changes in one generation during winter diapause imply strong selection and a relatively small number of overwintering eggs. During parthenogenetic reproduction the amount of variation remained stable or increased slightly, but deviations from the expected genotype frequencies increased toward autumn. Most of these deviations were due to a deficiency of heterozygotes. The analyses of evenness showed that clonal diversity remained relatively stable. The data demonstrate that selection changes continually in response to a changing environment and to the changing genetic organization of each population and, thus, maintains clonal diversity in $D$. magna populations.
\end{abstract}

\section{INTRODUCTION}

The environment of most populations changes from time to time and, presumably, so do the selective values of the different genotypes. The situation becomes particularly interesting if the selective values change direction between generations, which may maintain a polymorphic condition. Another type of selection that may be important in maintaining the genetic diversity of populations is frequency-dependent selection, whereby genotypes experience increased fitness as they become rare. In such populations where bisexuality is uncommon a third mechanism, heterozygote advantage, would not maintain genetic diversity, but rather cause the near-fixation of a multiply heterozygous clone (Berger, 1976).

Wright (1977) has pointed out that mass selection among populations consisting of a mixture of clones is more effective than in a panmictic bisexual population since selection acts on entire genotypes from generation to generation in the former and only on the additive genetic component of variance in the latter. Depending on migration and habitat differentiation, the result of clonal selection would either be extinction of all but the most fit clone or coexistence of clones in alternative niches. In fact, as revealed in several electrophoretic, cytological and tissue grafting studies, most parthenogenetic species are composed of multiple clones. Clues to the mechanisms of this clonal coexistence may be achieved by clarifying the distribution patterns of multiple clones in populations.

Daphnia magna is a cyclical parthenogen which has a life history consisting of a unisexual, apomictic phase, which is predominant during the favourable growing season and when the females produce diploid offspring in numerous parthenogenetic broods, and of a bisexual phase, which leads to the production of diploid resting eggs in a protective ephippium. The resting eggs persist throughout the unfavourable period, which may be winter or a dry season. Bisexuality affects the clonal structure through recombination by creating a greater spectrum of genotypes.

In this study genotype (clone) and allele frequencies were determined at several allozyme loci in ten intermittent $D$. magna populations for almost three years. The aim of the work was to find possible seasonal patterns in the genetic properties and clonal diversity of $D$. magna, and to consider the forces responsible for the changes or maintenance of the genetic structure. 


\section{MATERIALS AND METHODS}

Samples of ten Daphnia magna populations were collected from rock pools on four islets in the surroundings of Tvärminne Zoological Station in Hanko, southern Finland (Grid $27^{\circ} \mathrm{E}$ : 66:3) in years 1982-85. The 1985 samples included only the first sample of the reproductive season from each population. Populations Allgrundet A1, Allgrundet $\mathrm{B} 1$, Allgrundet $\mathrm{C}$ and Halsholmsgrunden were sampled every two or three weeks during the reproductive season. The other populations were sampled about once a month. The description of the populations is given in table 1 .

Using horizontal starch gel electrophoresis, populations (about 100 individuals from each sample) were screened for electrophoretic variants of the following enzyme systems which were found to be polymorphic and which had a relatively good resolution: Alkaline phosphatase (ALK), esterase (EST), glutamate oxaloacetate transaminase (GOT), leucine aminopeptidase (LAP) and malate dehydrogenase (MDH). Sampling and electrophoretic techniques employed have been described previously (Korpelainen, 1984). The gels were run for 5-6 hours at 100 volts (1982-83 samples) or for 4 hours at 200 volts (1984-85 samples). To record reproductive characteristics, about 200 adult sized individuals from each sample were screened for sex, mode of reproduction and number of eggs.

\section{RESULTS}

\section{Electrophoretic phenotypes}

EST-2 was the most variable locus of the six polymorphic loci studied. In all populations three alleles ( $\mathrm{F}, \mathrm{M}$ and $\mathrm{S}$ ) were found segregating, and all possible genotypes or at least most of them were detected in every population. The locus ALK-1 was relatively variable in Halsholmsgrunden but in the other populations it possessed only a low level of polymorphism. The number of alleles was two ( $\mathrm{F}$ and $\mathrm{M}$ ) or three (F, M and $\mathrm{S}$ ) except in the population Allgrundet A4 where a fourth and fastest allozyme, $Z$, was also found. The rare alleles $\mathrm{Z}, \mathrm{M}$ and $\mathrm{S}$ were detected mainly in heterozygous individuals.

All other loci possessed only low levels of heterozygosity. At the locus LAP-1 two (M and S or $\mathrm{F}$ and $\mathrm{M}$ ) or three alleles $(\mathrm{F}, \mathrm{M}$ and $\mathrm{S})$ were found segregating. At LAP-2 other populations had three alleles (F, M and S), except the population Allgrundet A4 which possessed two alleles, $M$ and S. At the locus GOT, in addition to the predominant allele $\mathrm{M}$, two rare alleles $\mathrm{F}$ and/or $\mathrm{S}$ were detected in all populations except in the monomorphic population Allgrundet A2. At the almost monomorphic locus $\mathrm{MDH}$ all three alleles ( $F, M$ and $S$ ) existed in all populations except Allgrundet A5 and Allgrundet B2 where the fast allozyme $\mathrm{F}$ was not found. Rare alleles, which existed in a particular population, were not detected every time in the samples examined.

\section{Population structure and dynamics}

All D. magna populations studied were intermittent, which means that in the beginning of each reproductive season populations were refounded from diapausing ephippial eggs. All or most of the eggs hatched into parthenogenetically reproducing females which initiated a rapid population growth. After the spring peak in the intensity of reproduc-

Table 1 Occurrence of Daphnia magna populations during the reproductive seasons $1982-84$ and the average size of population sites. No data available before July 1982.

\begin{tabular}{llllll}
\hline & & & & \multicolumn{2}{c}{ Occurrence } \\
\cline { 5 - 5 } Population & Area $\left(\mathrm{m}^{2}\right)$ & Depth $(\mathrm{cm})$ & 1982 & 1983 & 1984 \\
\hline 1. Allgrundet A1 & $2 \cdot 4$ & 1.5 & July-Sept & May-Aug & May-Aug \\
2. Allgrundet A2 & $1 \cdot 0$ & 15 & Aug-Oct & June-Sept & - $^{*}$ \\
3. Allgrundet A3 & $1 \cdot 4$ & 18 & Aug-Oct & May-Sept & May-Sept \\
4. Allgrundet A4 & $0 \cdot 3$ & 20 & Aug-Oct & June-July, Sept & May-Aug \\
5. Allgrundet A5 & $1 \cdot 0$ & 20 & Aug-Sept & May-Aug & June-July \\
6. Allgrundet A6 & $0 \cdot 8$ & 20 & Aug-Sept & June-Sept & May-Sept \\
7. Allgrundet B1 & $2 \cdot 6$ & 20 & July-Oct & May-Oct & May-July \\
8. Allgrundet B2 & $2 \cdot 6$ & 30 & Sept-Oct & May-Aug & May-Aug \\
9. Allgrundet C & $2 \cdot 2$ & 18 & Sept-Oct & May-Oct & May-Oct \\
10. Halsholmsgrunden & $40 \cdot 0$ & 50 & July-Sept & May-July & $-\dagger$ \\
\hline
\end{tabular}

\footnotetext{
* Population 2 used for a competition experiment in 1984, not included in this study after 1983.
}

$\dagger$ Population 10 did not exist in 1984. 
tion, the number of parthenogenetically reproducing females decreased along wth the average brood size. Bisexual females and males were observed regularly from August till October and only sporadically earlier in summer. Relatively high numbers of parthenogenetically or bisexually reproducing females throughout the reproductive seasons in most populations suggest that the populations had a good supply of food.

Population densities varied considerably in 1982-83. During those years densities were lowest in late July but rose suddenly in August. In 1984 population densities were more stable but several of the populations began to diapause as early as August. Low population densities and early diapause are suggested to be due to predation pressures, noxious algae composition or partial drying of the pools.

The intensity of reproduction, based on pooled data of May-June, July-August and SeptemberOctober samples, was measured as the average number of partherogenetic and sexual eggs per total number of adılt individuals in the sample (table 2). The number of ephippial eggs carried by bisexual females is only two at a time, which makes bisexual reproduction relatively unimportant for population growth before the end of the reproductive season when it is the major means of reproduction. The intensity of reproduction is highest early in the reproductive season when population densities are still low. Later the intensity decreases but has occasional late summer peaks as a consequence of lowered population densities or improved environmental conditions.

\section{Genotype and allele frequencies}

Significant temporal changes in both genotype and allele frequencies occurred during the almost three-year period of the study. Typical features of the changes were that there were no clear seasonal patterns and the fluctuations were more pronounced in genotype frequencies than in allele frequencies. Table 3 shows the frequency variation intervals of the most common genotypes and alleles at the polymorphic loci. During each reproductive season and between the seasons the frequencies changed rather rapidly and irregularly. A comparison of samples at the most variable locus EST-2 by the $G$ test revealed significant $(P<0.05)$ withinyear changes in genotype frequencies in 24 out of 27 cases and in allele frequencies in 19 out of 27 cases (table 4). In order to test between-year fluctuations, the genotype and allele frequencies of the late summer samples, which represent the stage when the intensity of reproduction was low and the number of bisexual females was still small, were compared to the late summer frequencies of the following year (1982 vs. 1983, 1983 vs. 1984) or spring frequencies were compared to the spring frequencies of the following year (1984 vs. 1985). Between-year genotype frequencies differed significantly in 12 out of 26 cases and allele frequencies in 12 out of 26 cases.

\section{The amount of heterozygosity}

Temporal changes in the amount of genetic variation were followed by computing the average expected heterozygosities for the pooled data of May-June, July-August and September-October samples, based on four polymorphic loci in 1982 (ALK-1, EST-2, GOT and MDH) and six loci in 1983-85 (the above loci and the loci LAP-1 and LAP-2) (table 5). In 1982-83 the amount of variation increased toward the autumn but in 1984 the heterozygosities were found to stay fairly stable. Throughout the summer continued

Table 2 Seasonal variation in the intensity of reproduction in 1982-85, based on pooled data of May-June, July-August and September-October samples

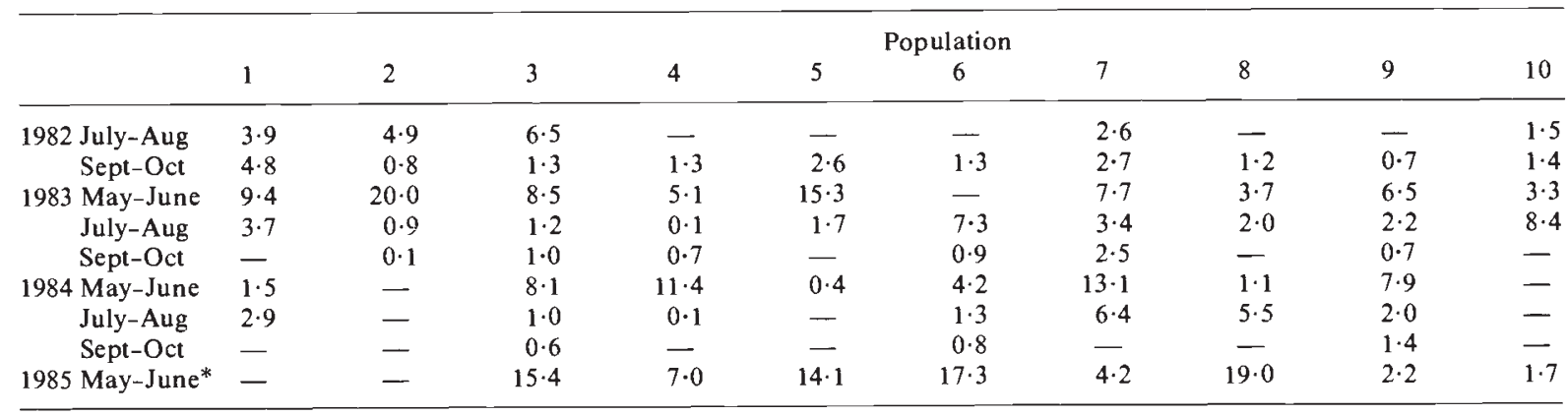

\footnotetext{
* Includes only the first sample of the season.
} 


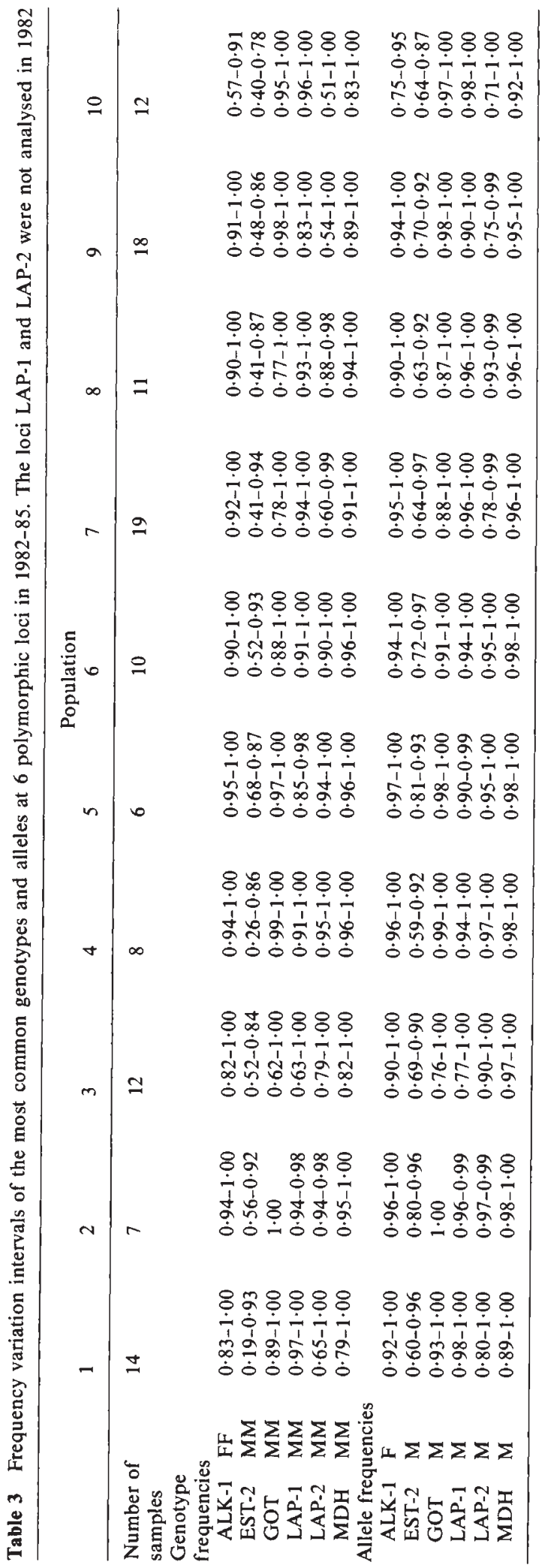




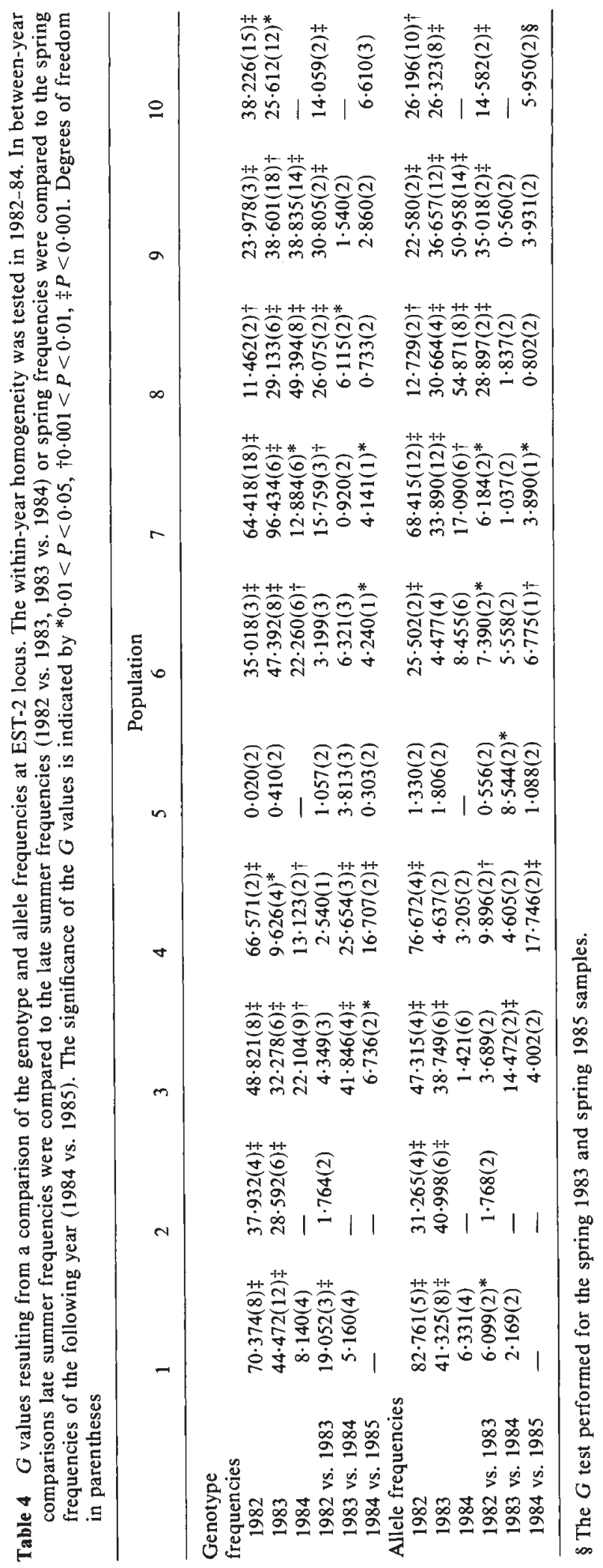




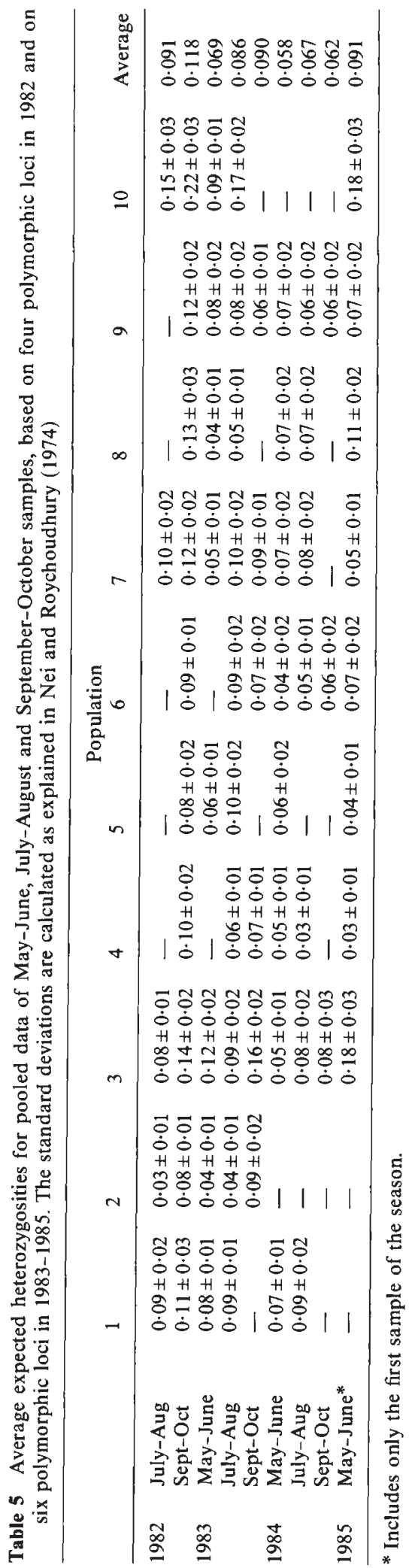


parthenogenetic reproduction did not suppress the amount of genetic variation.

Fixation indices, computed for all samples at the loci with the expected heterozygosity of $0 \cdot 10$ or more, were obtained from the equation $F=\left(H_{e}-H_{o}\right) / H_{e}$, where $H_{e}$ and $H_{o}$ are the expected and observed heterozygosities. Positive $F$ values indicate a deficiency and negative $F$ values an excess of heterozygotes. Whether the fixation ir dices departed significantly $(P<0.5)$ from zero was tested using a $\chi^{2}$ test ( $\mathrm{Li}$ and Horvitz, 1953). The genotype frequencies of spring populations were generally in good agreement with HardyWeinberg proportions but the deviations increased under continued parthenogenetic reproduction. 71 per cent of all samples were in agreement with the expected heterozygosity, but during the first third of the reproductive season the proportion was 88 per cent, during the second third 65 per cent and during the last third 61 per cent. 85 per cent of all significant deviations were due to a deficiency of heterozygotes.

\section{Association between the loci}

Apomictic parthenogenesis can maintain any array of genotypes at one locus as well as at several loci. In order to find potential associations between the loci, pairwise disequilibria were studied for locus pairs where the frequencies of the most common alleles were less than $0 \cdot 90$. At each locus the data were divided into a two allelic class: the most frequent allele was chosen as a principal allele and all other alleles were combined into a synthetic allele. This may result in some loss of power in testing for disequilibria (Weir and Cockerham, 1978), but the numbers of the rare alleles in the samples were not large enough for dealing with individual alleles.

The data of this study consisted of genotypes, and therefore Burrows' measure $\Delta$ was used (Cockerham and Weir, 1977). The use of this measure has been recommended by Weir (1979) in all cases where genotypic data are analysed. The maximum-likelihood estimate for $\Delta$ is obtained directly from genotypic data and is defined as follows (Cockerham and Weir, 1977):

$$
\tilde{\Delta}=\frac{N}{N-1}\left(\tilde{P}_{. .}^{i j}+\tilde{P}_{\cdot j}^{i \cdot}-2 \tilde{p}_{i} \tilde{q}_{j}\right),
$$

where tildes refer to maximum-likelihood estimates of allelic and gametic frequencies. The significance of $\Delta$ was tested by Fisher's $z$-transformation of the correlation coefficient, as described by Weir (1979).
Because the frequencies of the rare alleles at loci other than EST-2 were usually very low, the test of association could be performed only in 27 cases (ALK-1 vs. EST-2, ALK-1 vs. LAP-1, ALK-1 vs. LAP-2, EST-2 vs. GOT, EST-2 vs. LAP-1, EST-2 vs. LAP-2 or GOT vs. LAP-2). Significant disequilibrium was found in one case, in population 10 between the loci ALK-1 and EST-2 in July 1983. Although the small amount of data for the disequilibrium study limits the interpretation of the results, it seems that selection between different composite genotypes is not continuous enough to create significant associations between the loci.

\section{Clonal diversity and evenness}

The measures of clonal diversity and evenness are based on Simpson's (1949) species diversity index, $S I=\sum p_{i}^{2}$, where $p_{i}$ is the frequency of the $i$ th clone in the population and the summation is over all clones. This index gives the probability that two individuals selected at random will be of the same clone. It decreases as the distribution of individuals among the clones becomes more even and, thus, goes the "wrong" way as a measure of diversity. This is easily corrected by using $D=1-S I$. $S I$ is similar to some measures quantifying the amounts of genetic diversity (Parker, 1979). D, which is the complement of $S I$, is mathematically equivalent to sample heterozygosity or gene diversity at a single locus (Nei and Roychoudhury, 1974). The reciprocal of $S I$ is analogous to the effective number of alleles in a population (Kimura and Crow, 1964). If all clones were equally frequent, then the effective number of clones would equal the actual number. Otherwise, the effective number is always less than the actual number. To allow comparisons between populations with different sample sizes and numbers of clones, Fager (1972) suggested that the index should be scaled on the basis of the maximum range of values for a given sainple size and number of clones, when an appropriate measure would be

$$
E=\frac{D_{\mathrm{obs}}-D_{\min }}{D_{\max }-D_{\min }} .
$$

On this basis, 1 represents complete evenness $\left(D_{\text {obs }}=D_{\max }\right)$ and 0 extreme skewness $\left(D_{\text {obs }}=\right.$ $\left.D_{\min }\right)$.

The results of the examination of temporal dynamics in clonal diversity in D. magna populations are shown in table 6 . In reality, the numbers of clones are far greater than the numbers found by electrophoresis. Evenness and the number of 


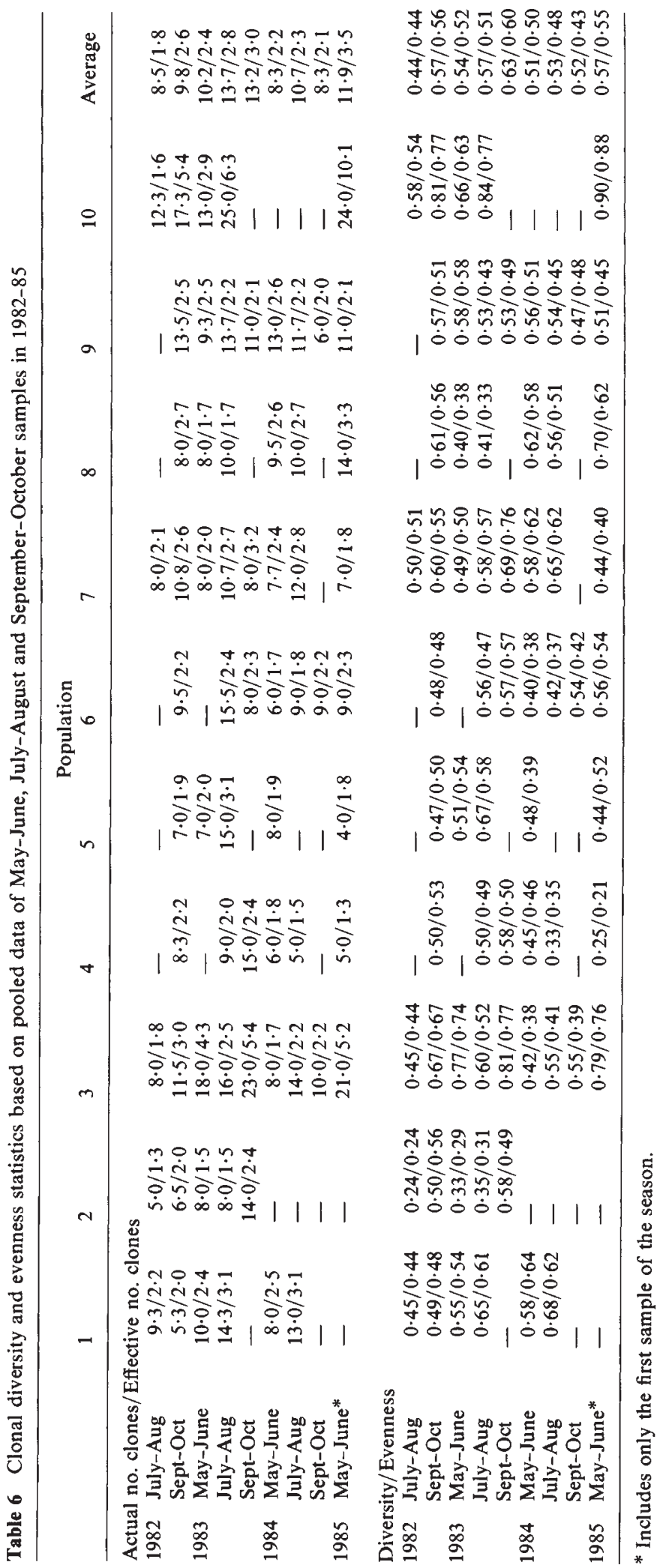


clones represent different aspects of clonal diversity, that is clonal equitability and richness, respectively (Hurlburt, 1971). Clonal diversity, as measured by the actual or effective number of clones, remained stable on the average even though changes within populations were more noticeable. Fluctuations in evenness were slight, in most cases also within populations. In non-pooled data, variation in clonal diversity measures was somewhat greater. Since clonal diversities stayed relatively stable while genotype frequencies fluctuated dramatically, this seems to indicate some selective mechanism protecting rare clones from extinction. A difficult problem, which must be considered in the interpretation of clonal diversity data in cyclically parthenogenetic $D$. magna populations, is bisexual recruitment which creates new clones into the system.

\section{DISCUSSION}

The effective population size influences the dynamics of genetic structure under natural selection since the probability and rate of fixation of a selectively advantageous mutant is an inverse function of effective size (Kimura and Ohta, 1971). Thus, smaller effective size is accompanied by a more rapid response to selection for the characters responsible for the fitness differential. The effective population size is a function of certain reproductive parameters as well as a function of systematic or random changes in population size. Under facultative apomixis, fecundity differences among unisexual clones may be greater than among bisexual genotypes because clonal fecundity will be effected by genotype, clonal growth rate and environmental conditions, which causes increasing variance in progeny numbers and reduction in the effective population size. On the other hand, unisexuality increases the generation time since the lives of individuals are extended. Then, also the effective size increases and causes a reduced response to selection in chronological time. Of particular importance is the fact that a population fluctuating about some given size has a smaller effective size than a population whose number is constant through time. Wright (1938) showed that if periodic reductions occur in the number of breeding individuals, the effective size is the harmonic mean over some time span. Since the harmonic mean is dominated by small numbers, cycles in population size greatly depress the effective size. It is noticeable that reduction in the number of breeding individuals does not have to be accompanied by a decline in population size.
Despite relatively large population sizes during most of the reproductive season, successive samplings from the Hanko populations of Daphnia magna revealed significant fluctuations in genotype and allele frequencies, but these changes did not show any clear seasonal pattern. It is obvious that rapid frequency changes reflect differences in fitness values between genotypic classes that are not ecological analogues. These selection pressures seem to be mainly unique for given populations and seasons because most of the changes were different in different populations. It is likely that the effect of selection is strengthened by the lowered effective population sizes due to great fecundity differentials and fluctuating population sizes. Specially great genetic changes in one generation during winter diapause imply strong selection, a fairly small number of overwintering eggs and some effect of genetic drift.

Temporal changes in the amount of genetic variation and in the degree of deviation of genotype frequencies from the expected proportions showed more seasonal regularity than genotype and allele frequencies. During the prolonged period of parthenogenetic reproduction the amount of variation remained stable or increased slightly. Parthenogenesis did not suppress the amount of genetic variation, and perhaps some ephippial eggs hatched after the beginning of the mainly parthenogenetic season. Deviations from the expected genotype frequencies increased toward autumn, which is the consequence of differences in all season sum fecundities between genotypes. In populations refounded from ephippial eggs, the genotype frequencies were in good agreement with Hardy-Weinberg expectations. A consistent tendency for excess heterozygosity at allozyme loci was not observed, as in many other D. magna studies (e.g. Hebert, 1974; Young, 1979). in fact, most of the genotypic deviations were due to a deficiency of heterozygotes.

Maintenance of genetic variation throughout the mainly parthenogenetic reproductive season indicates that cyclical parthenogenesis does not lead to a decrease of genetic variation. using phenotypic selection models directly coupled to the polygenic system, Lynch and Gabriel (1983) examined the consequences of periodic bisexuality. They found that by exploiting hidden genetic variance released by bisexuality, cyclical parthenogenesis periodically allows much higher rates of phenotypic evolution than can ever be attained under obligate bisexuality, and in the long run rates of phenotypic evolution are approximately independent of the frequency of bisexuality 
in populations which have attained their equilibrium levels of genetic variance. The periodic release of hidden genetic variance by the hatching of bisexually produced offspring allows a population of cyclical parthenogens to rapidly adjust to potentially new selective pressures. Lynch and Gabriel also suggest that cyclical parthenogenesis is rare not because any inherent disadvantages, but because of the strict requirements necessary for the transition to and maintenance of such a complex life cycle.

The analyses of evenness allowed an examination of the dynamics of clonal diversity from the standpoint of "community structure" of sympatric clones. Despite great frequency changes, there was no indication that selection could rapidly eliminate all but the single most fit clone. In fact, clonal diversity remained relatively stable. A major issue yet to be resolved is the mode of selection which maintains the diversity from generation to generation. The polymorphism of D. magna populations seems to be flexible, responding to physical and biological components of the environment by changes in genotype (clone) and allele frequencies. The data of the present study demonstrates that the intensity of selection, and even the direction, is often not the same in different stages. Selection changes continually in response to a changing environment and to the changing genetic organization of each population. Thus, the frequencies do not appear to be directed towards any single set of equilibrium frequencies. Rather, the enzyme polymorphism seems to resemble a "protected polymorphism", one in which selection acts so that genotypes (clones) and alleles are not lost, and in which the demography of selection may take various forms, including even continual movement. These variable fitness values, along with bisexual recruitment, may be essential in maintaining clonal diversity in temporary $D$. magna populations.

Acknowledgments I am grateful to Dr J. Lokki for comments on the manuscript, and to $\mathrm{K}$. Tankersley and $\mathrm{K}$. Vainio for valuable aid during the work. This study has been supported by grants from the Emil Aaltonen Foundation, the Alfred Kordelin Foundation and the Jenny and Antti Wihuri Foundation.

\section{REFERENCES}

BERGER, E. 1976. Heterosis and the maintenance of enzyme polymorphism. Am. Nat., 110, 823-839.

COCKERHAM, C. C. AND WEIR, B. S. 1977. Digenic descent measures for finite populations. Genet. Res., 30, 121-147.

FAGER, E. W. 1972. Diversity: A sampling study. Am. Nat., $106,293-310$

HERBERT, P. D. N. 1974. Enzyme variability in natural populations of Daphnia magna II. Genotypic frequencies in permanent populations. Genetics, 77, 323-334.

HURLBURT, S. H. 1971. The nonconcept of species diversity: A critique and alternative parameters. Ecology, 52, 577-586.

KIMURA, M. AND CROW, J. F. 1964. The number of alleles that can be maintained in a finite population. Genetics, 49 , 725-738.

KIMURA, M. AND OHTA, T. 1971. Theoretical Aspects of Population Genetics. Princeton Univ. Press, Princeton.

KORPELAINEN, H. 1984. Genic differentiation of Daphnia magna populations. Hereditas, 101, 209-216.

LI, C. C. AND HORVITZ, D. G. 1953. Some methods of estimating the inbreeding coefficient. Am. J. Hum. Genet., $5,107-117$.

LYNCH, M. AND GABRIEL, w. 1983. Phenotypic evolution and parthenogenesis. Am. Nat., 122, 745-764.

NEI, M. AND ROYCHOUdHuRY, A. K. 1974. Sampling variances of heterozygosity and genetic distance. Genetics, 76 , 379-390.

PARKER, E. D., Jr. 1979. Ecological implications of clonal diversity in parthenogenetic morphospecies. Am. Zool., 19, 753-762.

SIMPSON, E. H. 1949. Measurement of diversity. Nature, 163, 688.

WEIR, B. S. 1979. Inferences about linkage disequilibrium. Biometrics, 35, 235-254.

WEIR, B. S. AND COCKERHAM, C. C. 1978. Testing hypotheses about linkage disequilibrium multiple alleles. Genetics, 88, 633-642.

WRIGHT, S. 1938. Size of population and breeding structure in relation to evolution. Science, $87,430-431$.

WRIGHT, S. 1977. Evolution and the Genetics of Populations, vol. 3. Univ. Chicago Press, Chicago.

YOUNG, J. P. w. 1979. Enzyme polymorphism and cyclic parthenogenesis in Daphnia magna. I. Selection and clonal diversity. Genetics, 92, 953-970. 